\title{
Comparação de Técnicas de Fusão Aplicadas à Imagem Quickbird-2
}

\author{
Vagner Alex Pesck ${ }^{1}$, Attilio Antonio Disperati ${ }^{2}$ (in memorian), \\ João Roberto dos Santos ${ }^{3}$
}

${ }^{1}$ Programa de Pós-graduação em Ciências Florestais, Universidade Estadual do Centro-Oeste - UNICENTRO

${ }^{2}$ Departamento de Engenharia Florestal, Universidade Estadual do Centro-Oeste - UNICENTRO

${ }^{3}$ Instituto Nacional de Pesquisas Espaciais - INPE

\begin{abstract}
RESUMO
Este trabalho teve como objetivo testar quatro técnicas de fusão para melhoria da qualidade espacial da imagem Quickbird-2. As técnicas testadas foram: HSV e Brovey utilizando três bandas espectrais na composição colorido normal (3R2G1B), Gram-Schmidt e Principais Componentes utilizando as quatro bandas disponíveis na imagem. A área de estudo está inserida na Floresta Nacional de Irati, Estado do Paraná com 1600 ha. Analisando a qualidade visual das imagens fusionadas, os melhores resultados, em face de melhor conservação de cores, foram gerados pela técnica de Gram-Schmidt e Principais Componentes. Quanto à fidelidade espectral das imagens fusionadas (média e desvio padrão) com relação à multiespectral original, a técnica de Principais Componentes, preservou melhor o contraste da imagem original, mas a técnica de Gram-Schmidt forneceu melhor resultado quanto à conservação do brilho da imagem. Neste trabalho, pode-se concluir que qualquer uma das imagens fusionadas geradas pelas técnicas de Principais Componentes e Gram-Schmidt pode ser usada para atividade de mapeamento temático por gerar imagem de melhor qualidade em relação às demais técnicas testadas.
\end{abstract}

Palavras-chave: fidelidade espectral, fusão de imagens, Quickbird 2.

\section{Comparison of Fusion Techniques Applied Image Quickbird-2}

\begin{abstract}
This study aimed to test four fusion techniques in order to improve the spatial quality of the Quickbird-2 satellite image. The four techniques used were the following: HSV and Brovey, utilizing three spectral bands in a color composite (3R2G1B); Gram-Schmidt and Principal Components, using the four spectral bands available in the image. The study area was a part of the 'Irati' National Forest in the State of Parana, Brazil. The image used was from the Quickbird-2 satellite, with one panchromatic and four multi-spectral bands. Analyzing the visual quality of the fused images, the best results, due to better retention of color, were generated by the Gram-Schmidt and Principal Components techniques. As the spectral fidelity of the fused images concerns the original multi-spectral, the Principal Component technique preserved the contrast of the original image better, while the Gram-Schmidt technique provided better results concerning the preservation of image brightness. Any of the fused images generated by the Gram-Schmidt and Principal Components methods can be used for thematic mapping activity to generate better picture quality compared to the original image.
\end{abstract}

Keywords: spectral fidelity, image fusion, Quickbird 2. 


\section{INTRODUÇÃO}

Para melhor aproveitamento das informações produzidas por diferentes sensores, alguns métodos de processamento digital de imagens têm sido propostos (Ventura, 2002). Estas técnicas combinam imagens de diferentes características espectrais e espaciais para sintetizar uma nova imagem com melhor resolução espacial do que a imagem multiespectral original. A estas técnicas dá-se o nome de fusão de imagens.

Técnicas de fusão de dados são amplamente utilizadas na comunidade científica para explorar a potencialidade dos dados complementares (Pohl \& van Genderen, 1998). Isto é devido à quantidade crescente de dados multiespectrais adquiridos pelos novos sensores espaciais, tais como IKONOS-2 e Quickbird-2. A utilidade dos dados fusionados tem sido demonstrada em diversas aplicações ambientais, (Couloigner et al., 1998; Fanelli et al., 2001; Gonzáles Audícana \& Seco, 2002; Yang et al., 2000). Há uma variedade de técnicas de fusão, que têm sido propostas na literatura (Wang et al., 2005; Chavez Junior et al., 1991; Zhang, 2002), visando melhorar a qualidade (qualitativa e/ou quantitativa) das imagens de satélite. Por este fato, a qualidade resultante de uma imagem fusionada está relacionada a fatores espaciais, radiométricos e espectrais e, portanto, diferentes técnicas têm sido desenvolvidas visando objetivos diferentes.

A transformação Brovey consiste numa combinação aritmética entre uma imagem multiespectral de baixa resolução espacial representada no espaço RGB e uma imagem pancromática de alta resolução. No processo em questão, cada banda da imagem multiespectral é multiplicada pela razão da imagem pancromática dividida pela soma das bandas da imagem multiespectral (Vrabel, 1996). A fusão Brovey possui as mesmas limitações da IHS, pois trabalha com o sistema de cores RGB.

HSV é a sigla para hue, saturation e value, que significam, respectivamente, matiz, saturação e brilho. É um espaço de cor mais uniforme que o espaço RGB e que separa a cromaticidade da luminância. Matiz é a cor pura dominante percebida pelo observador. Saturação é o quanto a cor pura dominante (matiz) é diluída pela luz branca. Brilho (ou valor) é a quantidade de luz refletida (claro/ escuro), ou a quantidade de luz branca na cor (Nuñez, 2006). A transformação RGB-HSV separa a informação acromática (valor) e cromática (matiz e saturação) de uma imagem RGB (Schneider et al., 2003). No método de fusão pela transformada HSV, três bandas espectrais de baixa resolução espacial são transformadas do espaço RGB de cores para o espaço HSV. A componente $\mathrm{V}$ é substituída pela imagem PAN, e é efetuada a operação inversa, retornando para o espaço RGB (Schneider et al., 2003).

A fusão por Principais Componentes (PC) inicia-se com a transformação das bandas multiespectrais (sem limitação do número de bandas) em um mesmo número de componentes não correlacionadas. A primeira componente principal (CP1) é substituída pela banda pancromática que antes passa por um processo de equalização de média e variância para que fique a mais parecida possível com a CP1. Em seguida é realizada uma transformação inversa.

O método de Gram-Scmidt (GS), assim como o de Principais Componentes, é uma operação sobre vetores com o objetivo de torná-los ortogonais (maiores detalhes desta transformação podem ser encontrados em Smith, 2007). A fusão inicia-se com a simulação de uma banda pancromática a partir das bandas multiespectrais de baixa resolução espacial. Em sequência, a transformação de Gram-Schmidt é aplicada à banda pancromática simulada e às bandas multiespectrais, e a pancromática simulada é empregada como a primeira banda. Então, a primeira banda Gram-Schmidt é trocada pela banda pancromática de alta resolução e uma transformação inversa é aplicada para formar a imagem sintética de saída (RSI, 2009).

Os métodos de substituição de componentes (CS) ou fusão de imagens por substituição, tais como aqueles baseados em IHS (Carper et al., 1990; Edwards \& Davis, 1994; Liu, 2000; Tu et al., 2001), sobre a transformação Brovey (Gillespie, 1987) e na análise dos Componentes Principais (Schowengerdt, 1997), têm sido testados para melhorar a qualidade das imagens. Estas técnicas citadas acima são rápidas de serem processadas computacionalmente, resultam em bom desempenho espacial e são úteis para muitas 
tarefas de interpretação visual (Wang et al., 2005), mas os métodos PC e IHS são altamente sensíveis ao desalinhamento das bandas, como acontece para algumas imagens de alta resolução, tais como IKONOS-2 (Zhang, 2004). Por isso, nesse contexto, a técnica de Gram-Schmidt (GS) foi desenvolvida para melhorar a precisão dos métodos CS.

$\mathrm{O}$ presente trabalho teve como objetivo testar e avaliar as técnicas de fusão HSV, Brovey, Gram-Schmidt Spectral Sharpening e Principais Componentes aplicadas a imagens do sensor Quickbird-2 para identificação de alvos como vegetação estradas e agricultura, que posteriormente serão utilizadas para classificação da área de estudo.

\section{MATERIAL E MÉTODOS}

A área de estudo, com aproximadamente 600 ha, está inserida na Floresta Nacional de Irati (FLONA de Irati), nos municípios de Fernandes Pinheiro e Teixeira Soares, e a aproximadamente $150 \mathrm{~km}$ oeste de Curitiba - Estado do Paraná. Com área de 3.495 ha, a FLONA tem 36\% (1.272,9 ha) de floresta nativa com predominância de araucária e com o sub-bosque rico em espécies folhosas como Ilex paraguariensis, Ocotea porosa, Ocotea odorifera, Cedrela fissilis entre outras. Segundo a classificação climática de Köppen, a região tem clima do tipo $\mathrm{Cfb}$ com geadas frequentes no inverno. A temperatura média máxima é de $24,2{ }^{\circ} \mathrm{C}$ e a média mínima de $11,0^{\circ} \mathrm{C}$. A precipitação média mensal é de $193,97 \mathrm{~mm}$ e a umidade relativa média mensal do ar, de 79,58\%.

Foi utilizada a imagem Quickbird-2 (11bits) obtida em março de 2008, com resolução espacial de $60 \mathrm{~cm}$ no modo pancromático e $2,4 \mathrm{~m}$ no modo multiespectral e com quatro bandas multiespectrais: banda 1 (azul), banda 2 (verde), banda 3 (vermelho) e banda 4 (infravermelho próximo). A banda pancromática recobre todo o intervalo das bandas multiespectrais, fator importante na conservação de cores da imagem sintética (Fusionada). Para a realização dos testes foi utilizado o programa ENVI 4.6.1.

As bandas multiespectrais e a pancromática da imagem Quickbird-2 foram fusionadas com o objetivo de melhorar a resolução espacial das imagens. Para esse estudo, foram testadas quatro técnicas de fusão disponíveis no software ENVI 4.6.1:
HSV (três bandas), Color Normalized (Brovey) (três bandas), Gram-Schmidt Spectral Sharpening (quatro bandas) e Principais Componentes (quatro bandas). As bandas selecionadas para participarem da fusão foram as bandas 1, 2 e 3 para as fusões com 3 bandas e foi incluída a banda 4 (infravermelho próximo) nas fusões com 4 bandas.

Para analisar o resultado das fusões, foram realizados dois testes, sendo o primeiro uma análise visual entre os resultados das diversas técnicas de fusões utilizadas e a imagem multiespectral original. No segundo teste, foi verificada a fidelidade espectral das imagens fusionadas com relação à imagem multiespectral original. Como as imagens fusionadas possuem resoluções espaciais diferentes em relação à imagem original, não foi possível compará-las diretamente. Sendo assim, a imagem Quickbird-2 (pancromática e multiespectral) teve de ser reamostrada, de tal forma que as imagens fusionadas tivessem a mesma resolução espacial da imagem multiespectral original. A fusão entre as imagens degradadas é considerada uma fusão simulada somente para efeito de avaliação. No presente estudo, a imagem pancromática original foi degradada para resolução de $2,4 \mathrm{~m}$ e a multiespectral original para 9,6 m. A fusão entre essas duas imagens gerou uma nova imagem fusionada com resolução de $2,4 \mathrm{~m}$. Para a comparação das imagens, foram utilizadas a média, desvio padrão e correlação entre bandas. Para efeito de comparação entre as bandas da imagem original e das fusionadas, os histogramas de cada imagem foram plotados para melhor visualização.

\section{RESULTADOS E DISCUSSÃO}

Podemos analisar na Figura 1 o resultado das técnicas de fusão testadas neste presente trabalho.

Em todas as imagens fusionadas neste presente trabalho, foi possível ver o ganho em detalhes, como estradas mais visíveis e, principalmente, a vegetação ficou com melhor definição.

Após a aplicação das técnicas de fusão na imagem Quickbird-2, os resultados mostraram visualmente melhor conservação de cores em Principais Componentes e Gram-Schmidt, empregando as 4 bandas multiespectrais (Figura $2 \mathrm{~b}$ e $2 \mathrm{e}$ ). Todas as técnicas testadas produziram imagens fusionadas 


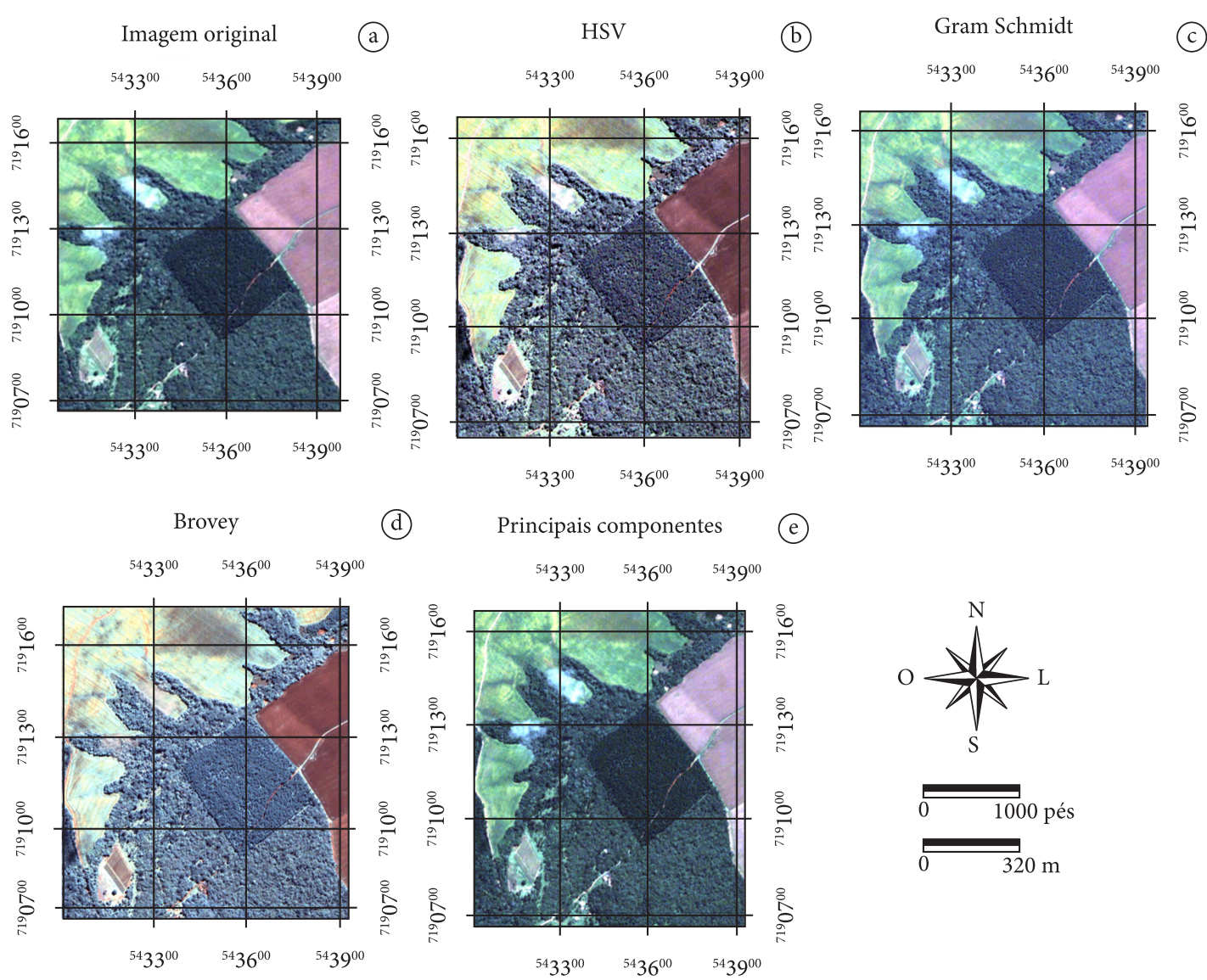

Figura 1. Comparação entre a imagem multiespectral original (a) e as técnicas de fusão testadas.

Figure 1. Comparison between the original multispectral image (a) and fusion techniques tested.

com uma variação de cores bastante significativa em comparação com a imagem original, destacando-se as fusões $H S V$ e Brovey que apresentaram, nas áreas com vegetação, cores variando do verde brilhante até o azul escuro. Nas técnicas que utilizaram três e quatro bandas, verificou-se uma diferença de tonalidade também para as áreas cobertas com vegetação. Nas fusões com três bandas, a cor da vegetação ficou com tom verde mais brilhante.

Estes dois problemas detectados acima são decorrentes da amplitude espectral da imagem pancromática do sensor Quickbird-2, que se estende até o infravermelho próximo, região do espectro onde a vegetação possui resposta muito alta (Pinho et al., 2005). Desta forma, processos de fusão que utilizam as imagens do referido sensor devem incorporar a banda 4 (infravermelho) para que não apresentem grandes mudanças de cores. $\mathrm{O}$ mesmo autor também encontrou que, em relação à conservação de cores, o melhor resultado foi obtido pela técnica de Principais Componentes.

Luz (2009), utilizando o sensor ALOS Palsar, verificou que as fusões pelo método Gram-Schmidt apresentaram as cores mais vibrantes, principalmente na operação que utilizou as quatro bandas. O método Brovey apresentou cores semelhantes ao método Gram-Schmidt com quatro bandas, apresentando tons azulados por toda a imagem, o que não pode ser verificado neste trabalho, sendo que as fusões que apresentaram mais semelhanças foram às técnicas de Gram-Schmidt e Principais Componentes.

Santurri et al. (2010) estudaram a qualidade das imagens fusionadas para mapear o deslizamento de terra. A avaliação foi feita através de uma escala de detecção baseada em interpretação qualitativa da imagem, com base em cinco níveis de classificação, 
sendo: 5 (insuficiente), 4 (mau), 3 (médio), 2 (bom), 1 (excelente) para as classes de textura, cor e contraste. Para estes autores, os métodos de Principais Componentes e o de Gram-Schmidt forneceram os melhores resultados no contexto da detecção de deslizamento de terra entre os métodos de fusão testados.

No segundo teste, foi analisada a fidelidade espectral das imagens fusionadas com relação à multiespectral original. Na Tabela 1, são apresentados os valores de média dos pixels, desvio padrão e correlação para a imagem multiespectral e para as fusões.

Pode-se observar que a média e o desvio padrão do método HSV se mostraram bem diferentes dos valores da imagem original. A média e o desvio padrão estão relacionados com o brilho e contraste da imagem, respectivamente (Luz, 2009). Havendo uma menor variação na média, o brilho é conservado.
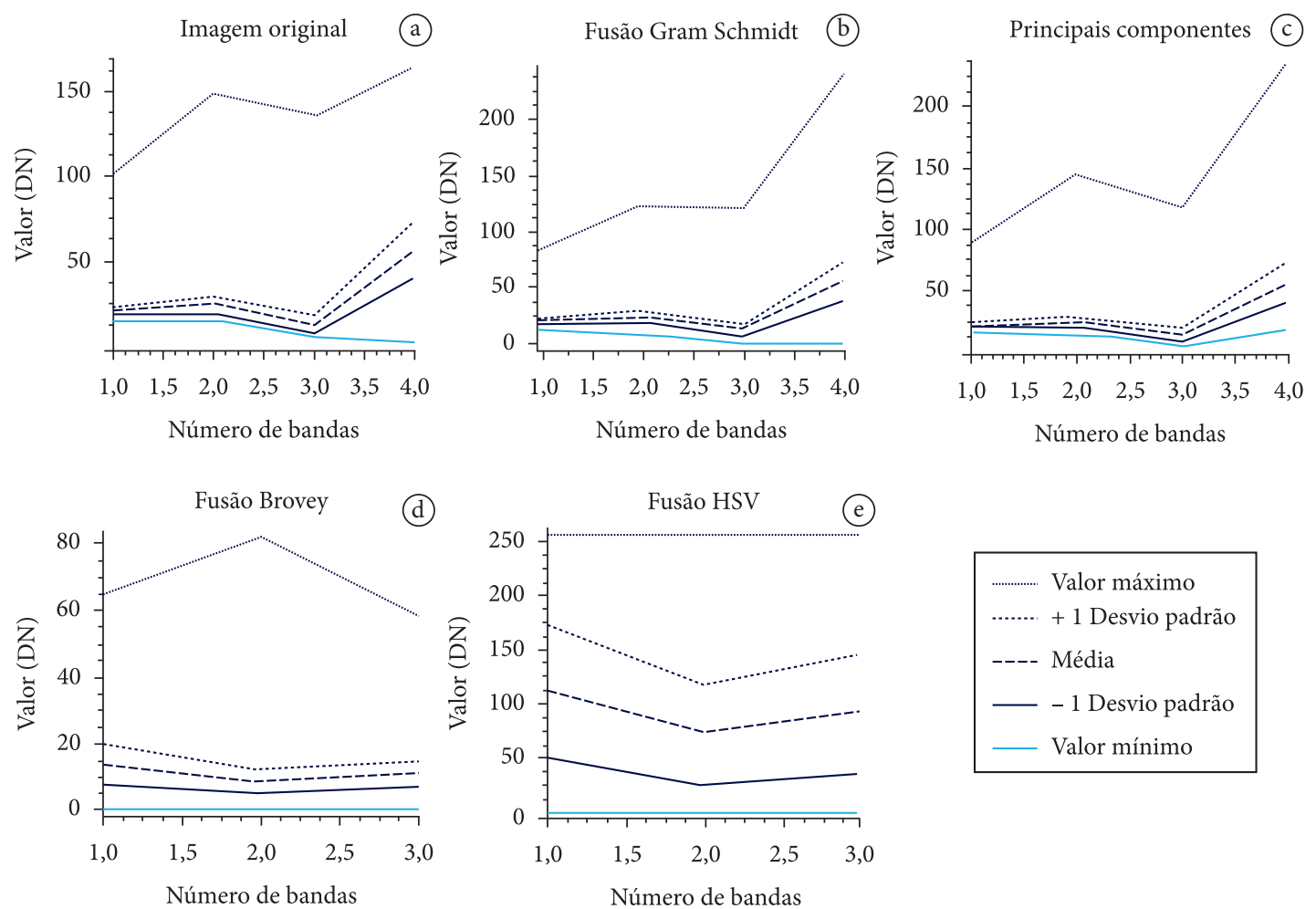

Figura 2. Histogramas comparando imagem original e imagem simulada.

Figure 2. Histograms comparing the original image and image simulated.

Tabela 1. Comparação entre os parâmetros estatísticos da imagem multiespectral original e da sintética simulada.

Table 1. Comparison between statistical parameters of the original multispectral image and the synthetic simulated.

\begin{tabular}{|c|c|c|c|c|c|c|c|c|c|c|c|c|c|}
\hline \multirow{2}{*}{ Método } & \multicolumn{3}{|c|}{ Banda 1} & \multicolumn{3}{|c|}{ Banda 2} & \multicolumn{3}{|c|}{ Banda 3} & \multicolumn{3}{|c|}{ Banda 4} & \multirow{2}{*}{ Média } \\
\hline & Média & DP & Cor. & Média & DP & Cor. & Média & DP & Cor. & Média & DP & Cor. & \\
\hline Orig. & 19,7 & 1,9 & 1 & 24,1 & 4,8 & 1 & 12,2 & 5,4 & 1 & 56,3 & 16,6 & 1 & 1,00 \\
\hline HSV & 111,4 & 60,7 & 0,75 & 72,8 & 46,8 & 0,92 & 90,6 & 54,2 & 0,92 & - & - & - & 0,92 \\
\hline Brovey & 13,5 & 5,6 & 0,37 & 8,4 & 3,7 & 0,75 & 10,6 & 3,8 & 0,75 & - & - & - & 0,75 \\
\hline GS & 19,7 & 1,9 & 0,92 & 24,2 & 4,8 & 0,92 & 12,3 & 5,6 & 0,93 & 56,1 & 16,9 & 0,39 & 0,93 \\
\hline $\mathrm{PC}$ & 19,7 & 2,0 & 0,95 & 24,4 & 5,1 & 0,95 & 12,4 & 5,8 & 0,95 & 56,8 & 14,5 & 0,55 & 0,95 \\
\hline
\end{tabular}

Orig. = imagem original; GS = Gram Schmidt; DP = desvio padrão; PC = principais componentes; Cor. = correlação. 
Uma menor variação entre os valores de desvio padrão demonstra melhor preservação do contraste da imagem (Pinho et al., 2005). Por outro lado, a técnica de fusão HSV teve alta correlação entre as bandas devido à banda pancromática apresentar um intervalo espectral que engloba o intervalo das três bandas multiespectrais de entrada. Segundo Fuckner (2007), a fusão pela técnica IHS apresenta bons resultados quanto à representação de cores somente quando a imagem pancromática apresenta um intervalo espectral que engloba o intervalo das três bandas multiespectrais de entrada. Apesar de o sistema de cores HSV não ser o mesmo do IHS, o princípio da técnica de fusão é o mesmo. Luz (2009) obteve baixa correlação $(0,318)$ testando esta técnica por utilizar a composição falsa cor devido a banda 4 (infravermelho próximo) englobar apenas uma pequena parte da banda pancromática, sendo que, neste trabalho, esta mesma técnica obteve correlação de 0,92 , sendo ligeiramente maior que o resultado obtido por Luz (2009).

$\mathrm{Na}$ fusão, a técnica Principais Componentes resultou em uma menor variação entre os valores de desvio padrão, ou seja, preservou-se melhor o contraste da imagem original. Já a técnica de Gram-Schmidt forneceu melhores resultados para o valor média, indicando uma conservação do brilho da imagem.

A comparação entre os histogramas (imagem original / imagem simulada) também revelou resultados interessantes como mostra a Figura 2.

Novamente o melhor desempenho foi obtido pelas técnicas Principais Componentes e Gram-Schmidt, sendo que a primeira apresentou histogramas mais coerentes com a imagem original.

Com base nos dois testes efetuados, podemos verificar que as técnicas de Gram-Schmidt e Principais Componentes se mostraram superiores às demais técnicas (HSV e Brovey) testadas neste presente estudo.

\section{CONCLUSÕES}

Considerando a qualidade visual das imagens fusionadas e, principalmente, devido à melhor conservação das cores, as técnicas de Gram-Schmidt e Principais Componentes forneceram os melhores resultados. Em relação à fidelidade espectral das imagens fusionadas relativas à multiespectral original, a técnica de fusão Principais Componentes preservou melhor o contraste da imagem original, mas a técnica de Gram-Schmidt forneceu melhor resultado quanto à conservação do brilho da imagem.

A imagem Quickbird-2 se mostrou eficiente para a identificação de alvos, sendo as técnicas de Principais Componentes e Gram-Schmidt ligeiramente superiores às demais técnicas testadas.

Recomenda-se testar novas técnicas de fusão e a utilização de outros sensores como Worldview-2 e Geoeye, inserindo novos tipos de alvos como áreas urbanas, reflorestamentos, corpos de água, etc.

\section{AGRADECIMENTOS}

Os autores agradecem: à Coordenação de Aperfeiçoamento de Pessoal de Nível Superior (CAPES); à Universidade Estadual do Centro Oeste do Paraná (UNICENTRO); o suporte técnico à SULSOFT; e ao Prof. Dr. Afonso Figueiredo Filho a aquisição da imagem, que, juntamente, contribuíram para a realização deste projeto.

\section{STATUS DA SUBMISSÃO}

Recebido: 02/12/2010

Aceito: 20/06/2011

Resumo publicado online: 25/06/2011

Artigo completo publicado: 30/06/2011

\section{AUTOR(ES) PARA CORRESPONDÊNCIA}

\section{Vagner Alex Pesck}

Programa de Pós-graduação em Ciências Florestais, Universidade Estadual do CentroOeste - UNICENTRO, PR-153, Km 7, Bairro Riozinho, CEP 84500-000, Irati, PR, Brazil e-mail: vagneralexp@yahoo.com.br

\section{Attilio Antonio Disperati (in memorian)}

Departamento de Engenharia Florestal, Universidade Estadual do Centro-Oeste UNICENTRO, PR-153, Km 7, Bairro Riozinho, CEP 84500-000, Irati, PR, Brazil e-mail: disperati@avalon.sul.com.br 


\section{REFERENNCIAS}

Carper WJ, Lillesand TM, Kiefer, RW. The use of intensity- hue-saturation transformations for merging SPOT panchromatic and multispectral image data. Photogrammetric Engineering \& Remote Sensing 1990; 56:459-467.

Chavez Junior PS, Sides SC, Anderson JA. Comparison of three different methods to merge multiresolution and multispectral data: Landsat TM and SPOT panchromatic. Photogrammetric Engineering \& Remote Sensing 1991; 57(3):295-303.

Couloigner I, Ranchin T, Valtonen VP, Wald L. Benefit of the future SPOT-5 and of data fusion to urban road mapping. International Journal of Remote Sensing 1998; 19(8):1519-1532. http://dx.doi. org/10.1080/014311698215324

Edwards K, Davis PA. The use of Intensity-HueSaturation transformation for producing color shadedrelief images. Photogrammetric Engineering \& Remote Sensing 1994; 60(11):1369-1374.

Fanelli A, Leo A, Ferri M. Remote sensing images data fusion: A wavelet transform approach for urban analysis. In: Proceedings of the Joint IEEE/ISPRS Workshop Remote Sens. Data Fusion Over Urban Areas; 2001; Rome. Rome: University of Rome; 2001. p. 112-116.

Fuckner MA, Rosa VGC, Rosemback R. Análise comparativa da aplicação de transformações radiométricas e técnicas de fusão de imagens em área urbana. In: Anais do XIII Simpósio Brasileiro de Sensoriamento Remoto; 2007; Florianópolis. São José dos Campos: INPE; 2007. p. 6343-6350.

Gillespie AR, Kahle AB, Walker RE. Color enhancement of highly correlated images-II. Channel ratio and 'chromaticity' transformation techniques, Remote Sensing of Environment 1987; 22(3): 343-365.

Gonzáles Audícana M, Seco A. Fusion of multispectral and panchromatic images using wavelet transform. Evaluation of crop classification accuracy. In: Proceedings of the XXII EARSeL Annual Symposium "Geoinformation for European-wide integration"; 2003; Prague, Czech Republic. Prague ; 2003. p. 265-272.

Liu JG. Smoothing filter-based intensity modulation: A spectral preserve image fusion technique for improving spatial details. International Journal of Remote Sensing 2000; 21(18):3461-3472. http://dx.doi. org/10.1080/014311600750037499
Luz J. Imagens Alos para o Mapeamento da Vegetação Arbórea e Outros Usos do Solo em Área de Floresta Ombrófila Mista [dissertação]. Curitiba: Universidade Federal do Paraná; 2009.121 p.

Nuñez JR. Segmentação automática e classificação em vídeos esportivos [dissertação]. Curitiba: Pontifícia Universidade Católica do Paraná; 2006. 178 p.

Pinho CMD, Rennó CD, Kux HJH. Avaliação de técnicas de fusão aplicadas à imagem Quickbird. In: Anais do XII Simpósio Brasileiro de Sensoriamento Remoto; 2005; Goiânia, Brasil. RBC; 2005. p. 4225-4232.

Pohl C, van Genderen JL. Multisensor image fusion in remote sensing: concepts, methods and applications. International Journal of Remote Sensing 1998; 19:823-854. http://dx.doi.org/10.1080/014311698215748

Research Systems Inc. - RSI. Tutorial Envi FX Classificação por regras-segmentação orientada ao objeto; $2009.15 \mathrm{p}$

Schneider MJ, Bellon ORP, Araki H. Experimentos em fusão de imagens de alta resolução. Boletim de Ciências Geodésicas 9(1):75-88, 2003.

Schowengerdt RA. Spectral Transforms. In: Schowengerdt RA. Remote Sensing: Models and Methods for Image Processing. London: Academic Press; 1997. $522 \mathrm{p}$.

Santurri L, Carlà R, Fiorucci F, Aiazzi B, Baronti S, Cardinali M, Mondini A. Assessment of very high resolution satellite data fusion techniques for landslide recognition. In: Wagner W, Székely B, editor. ISPRS TC VII Symposium - 100 Years. ISPR: Viena; 2010.

Smith JO. Gram-Schmidt Orthogonalization. In: Smith JO. Mathematics of discrete Forier transforms (DTF), with Audio Applications; [online]. 2007. [cited 2011]. Available from: https://ccrma.stanford.edu/ jos/dft/ Gram_Schmidt_Orthogonalization.html\#11780

Tu T-M, Su S-C, Shyu H-C, Huang PS. A new look at IHS-like image fusion methods. Information Fusion 2001; 2:177-186. http://dx.doi.org/10.1016/S15662535(01)00036-7

Ventura FN. Fusão de imagens de sensores remotos utilizando a transformação wavelet [dissertação]. São José dos Campos: Instituto Nacional de pesquisas Espaciais; 2002.

Vrabel J. Multispectral imagery band sharpening study. Photogrammetric Engineering \& Remote Sensing 1996; 62(9): 1075-1083.

Wang Z, Ziou D, Armenakis C, Li D, Li Q. A comparative analysis of image fusion methods. IEEE Transactions On 
Geoscience And Remote Sensing 2005; 43(6):1391-1402. http://dx.doi.org/10.1109/TGRS.2005.846874

Yang W, Cauneau F, Paris J-P, Ranchin T. Influence of landscape changes on the results of the fusion of $\mathrm{P}$ and XS images by different methods. In: Proceedings III Conference Fusion of Earth Data: Merging Point Measurements, Raster Maps Remotely Sensed Images; 2000; Sophia Antipolis. European Association of Remote Sensing Laboratoriesp; 2000. 47-56.
Zhang Y. Problems in the fusion of commercial highresolution satellite images as well as Landsat 7 images and initial solutions. In: Geospatial Theory, Processing and Applications: Proceedings of the ISPRS Commission IV Symp; 2002; Ottawa. Ottawa: Canadian Institute of Geomatics; 2002.

Zhang Y. Understanding image fusion. Photogrammetric Engineering \& Remote Sensing 2004; 70(6):657-661. 